\title{
Hard X-ray tomographic spectroscopy in TCV
}

\author{
S. Coda, J. Kamleitner, S. Gnesin, Ph. Marmillod \\ Centre de Recherches en Physique des Plasmas, Ecole Polytechnique Fédérale de Lausanne \\ (EPFL-CRPP), 1015 Lausanne, Switzerland \\ E-mail: Stefano.Coda@epfl.ch
}

A novel, 3-camera tomographic hard X-ray (HXR) spectrometer has been designed, assembled, installed and operated on the TCV tokamak for the study of suprathermal electrons. A fourth camera is planned for the near future to complete the set.

HXR detection is based on room-temperature CdTe technology. Each of the cameras comprises 24 active detectors plus one "blind", heavily shielded detector to discriminate against uncollimated gamma-ray radiation. An individual collimator arrangement for each chord provides total étendue control. The high incidence of very high energy X-and $\gamma$-ray emission from runaway electrons in TCV, due to the often low operational density, has necessitated sizable shielding, amounting to at least $2 \mathrm{~cm}$ of tungsten between each detector and the TCV vessel. Up to 36 different absorber combinations can be selected in front of the detectors, providing a wide variety of high-pass energy filters for compatibility with any emission level. The two lateral cameras feature the option of rotating on their axes, shifting the chord fan from poloidal to toroidal.

The electronic pulse processing chain, primarily based on charge integration, was developed entirely in-house, resulting in a high-performance system with a potential maximum detectable photon rate of $\sim 6 \times 10^{5} / \mathrm{s}$. Digital acquisition at $12 \mathrm{MSamples} / \mathrm{s}$ provides the full pulse history. Pulse height analysis is thus effected by digital postprocessing. The cameras feature a spatial resolution of approximately $8-10 \%$ of the minor radius and an energy resolution of $\sim 7 \mathrm{keV}$ in the 10-300 keV range.

The diagnostic has been employed fruitfully in a variety of settings. The first tomographically reconstructed HXR emission images in a non-circular plasma have been generated, addressing the physics of the interaction of ECRH waves with the plasma - including the resulting poloidal and toroidal asymmetries - in concert with numerical Fokker-Planck modeling aided by a synthetic diagnostic. The interaction of various types of MHD instabilities with suprathermal electrons has also been studied.

First EPS Conference on Plasma Diagnostics - 1st ECPD,

14-17 April 2015

Villa Mondragone, Frascati (Rome) Italy 


\section{Introduction}

The detection of hard X-ray (HXR) bremsstrahlung emission is a prime technique for studying the high-energy tail of the electron distribution function (e.d.f.) in a thermonuclear plasma. Studying this tail is of particular interest when the e.d.f. is non-Maxwellian. Significant suprathermal electron populations can be generated by external means, typically by injection of electromagnetic waves, and can also be self-generated by, e.g., magnetohydrodynamic (MHD) instabilities. In tokamaks in particular, resistive MHD modes originating at the $q=1$ and $q=2$ surfaces, associated typically with the sawtooth crash and disruptive events, respectively, often accelerate electrons to suprathermal energies. The study of this population is then important to elucidate the physics that underlies their generation [1].

In the TCV tokamak [2], strong motivation for suprathermal electron studies is provided by an electron cyclotron resonance heating and current drive (ECRH and ECCD) system of unusually high power density (4.5 MW at its peak) [3]. The physics of ECRH and particularly ECCD is mediated by the suprathermal population. Extensive HXR measurements were performed in the past using a single multi-channel camera on loan from CEA-Cadarache [4]. The assumption of poloidal emission uniformity, necessary to invert data from a single camera, is known to be valid only in a very approximate sense. A tomographic system is then necessary to resolve the full $2 \mathrm{D}$ poloidal distribution. Such a system has now been developed for TCV, comprising three cameras (with a fourth one planned), and utilizing state-of-the-art technology and methods [5, 6, 7, 8]. This constitutes the first system of its kind applied to a noncircular tokamak plasma.

\section{Design process}

Every step in the design of the TCV HXR diagnostic went through some form of optimization $[5,9]$. It is particularly challenging to find an optimal distribution of viewpoints and lines of sight in a device with the flexibility of TCV, where plasma configurations and positions can be varied at will within the confines of the vacuum vessel. The feat was accomplished with the assistance of modeling tools, namely the LUKE quasilinear Fokker-Planck code [10] combined with the R5X2 HXR synthetic diagnostic [11]. A set of representative ghost emission patterns and of plasma configurations were selected, and numerical simulations were performed to ultimately produce the signal distribution in the putative diagnostic; then, a tomographic inversion was performed and the result compared with the original ghost. These emission patterns ranged from poloidally-symmetric emissivity profiles reconstructed from past, non-tomographic HXR measurements during ECRH in $\mathrm{TCV}$, to periodic poloidal distributions with a poloidal wave number $m$, to simulate increasingly complex poloidal asymmetries. A "Reconstruction Variance" in particular was defined to quantify the goodness of the match, simply consisting of the normalized squared cumulative discrepancy between ghost and reconstructed image. On this basis it was concluded that a set of four cameras (one at the top, one at the bottom, and two on the side; see Fig. 1a) was a suitable compromise. Indeed, as shown by Fig. 1b, improvements level off beyond four cameras, while costs of course increase. Every other aspect of the geometrical arrangement was subject to the same procedure, including the angular orientation, the distance of the detectors from the plasma, and the number of detectors per camera. The latter is of course dependent on the smallest spatial structures that need 

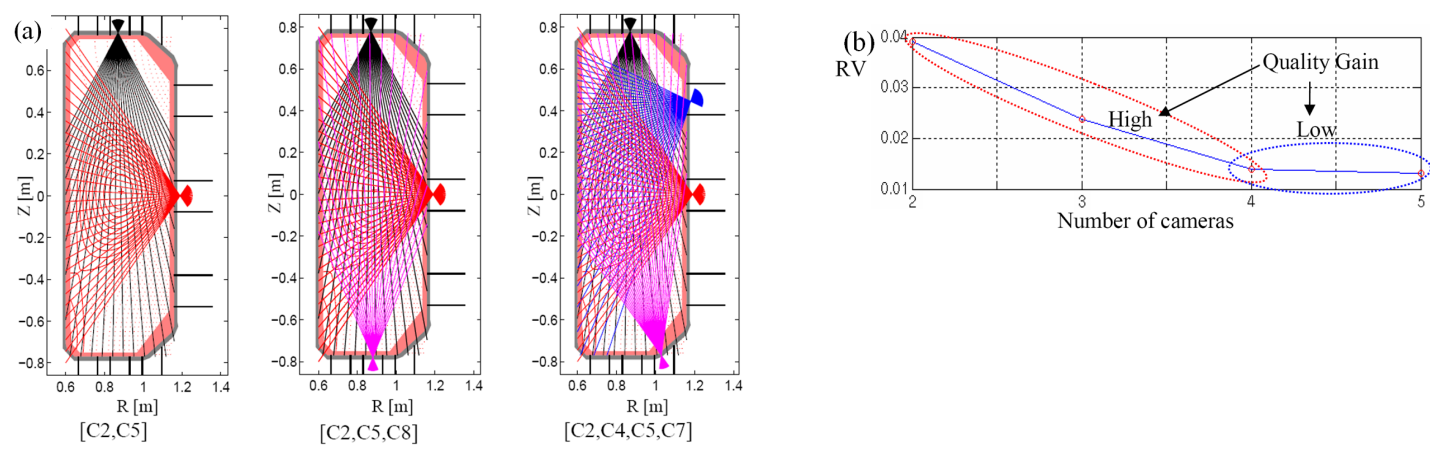

Figure 1: (a) Lines of sight of optimal configurations with 2, 3 and 4 cameras, the latter being the one finally chosen; (b) Reconstruction Variance (RV) as a function of the number of cameras.

to be resolved: past data suggested that a spatial resolution and sampling of $\sim 8 \%$ of the minor radius was adequate. This resulted in 24 lines of sight per camera [5, 6, 9].

The choice of the detectors was equally complex. Room-temperature, solid-state detectors were quickly selected because of their compactness - due in great part to the lack of cooling equipment and magnetic shielding - and ease of operation. Several options remained, however, and we undertook a rigorous series of tests on a number of commercial options, complete with logging over long time scales to monitor long-term stability. In particular, CZT and CdTe detectors were examined, while an HgI2 option was quickly abandoned because of insufficient maturity and reliability. The CdTe detectors tested featured either Ohmic or Schottky contacts. A thickness of $2 \mathrm{~mm}$ was dictated by the requirement of a sufficiently broad energy range (up to $\sim 300 \mathrm{keV}$ ). The performance of Schottky detectors was superior a priori, featuring a narrower photopeak and the smallest level of tailing (Fig. 2); however, severe polarization effects (worsened by thickness) rendered the detector performance erratic, and long-term stability was exceedingly poor. Ohmic-type CdTe detectors were then preferred. The final choice was a cubic detector of $2 \mathrm{~mm}$ side [6].

Collimation is crucial for spatial resolution and high-pass filtering. The most common choice is a pinhole collimator common to a set of lines of sight. This inevitably results in obligatory variations of étendue among lines of sight, even after a design optimization. To overcome this limitation, a variant of the Soller type of collimator was introduced: so-called "radial" Soller collimators essentially provide each line of sight with its own collimator, while ensuring an optimal use of space. In particular, the choice of "diverging" collimators, with lines of sight crossing at a virtual fulcrum, maximizes the space available for detectors and their associated electronics and support units, without sacrificing compactness (Fig. 3). Tungsten was chosen as collimator material, as it provides the highest stopping power of any material, with few exotic or costly exceptions (Au, Pt).

\section{Description and characteristics}

The diagnostic already comprises three cameras, with a fourth one already designed and currently under construction. Each camera comprises 24 active detectors. An additional, 25th detector is hidden behind the collimator material, with no direct view of the plasma; this "blind" detector is used for discrimination against very high-energy, uncollimated radiation, typically coming from runaway electrons. To protect the primary vacuum interface, all detectors and preamplifier cards are located in a differentially-pumped rough vacuum. 


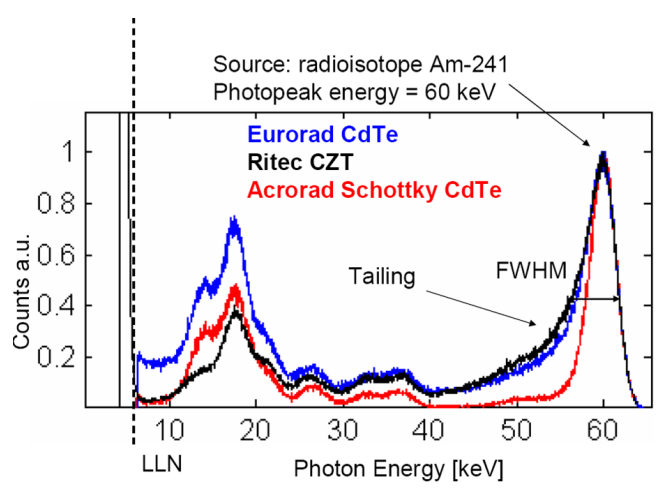

Figure 2: Pulse height analysis of photons from a monochromatic Am-241 source (60 keV) using three different types of detectors. The Eurorad and Ritec detector have Ohmic contacts.
The spatial resolution is approximately $2 \mathrm{~cm}$ near the plasma center (the plasma minor radius being $\sim 25 \mathrm{~cm}$ ). The energy resolution is determined primarily by the detector properties and is in the order of $7 \mathrm{keV}$ throughout the spectrum; while cooled detectors provide better resolution, the essentially broadband nature of bremsstrahlung radiation made it unwise to sacrifice the compactness afforded by roomtemperature detectors. Radiation from $\sim 13$ to $300 \mathrm{keV}$ can be detected. A key parameter is the maximum count rate, ultimately limited by the pile-up effect related to the finite rise time of the charge collection in the crystal; an optimized choice of pulse detection technique brings the maximum rate to $\sim 6 \times 10^{5}$ counts/s. With the chosen étendue, this is sufficiently high to require no special high-pass filtering (i.e., absorber filters) except at high ECRH power [6, 7, 8].

The integrated and amplified charge signal is acquired at 12 Msamples/s, so that the entire pulse history is recorded and pulse height analysis is performed by post-processing software, with no a priori binning limitations. The time resolution of the measurement is determined by the photon statistics themselves. A required statistical signalto-noise ratio of 10 generally limits the time resolution to $\sim 5 \mathrm{~ms}$. However, in the presence of

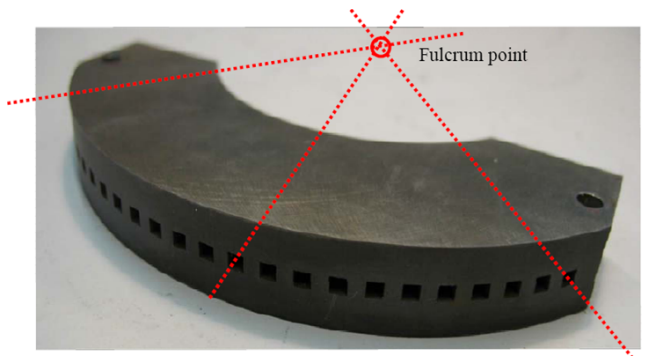

Figure 3: Radial tungsten collimator. periodic or at least repetitive events, such as sawtooth oscillations, conditional averaging (i.e., averaging over multiple, appropriately time-shifted events: e.g., using each sawtooth crash time as the reference time), can be usefully applied to increase the effective resolution, to the order of even $\sim 10 \mu \mathrm{s}$ in the case of especially high-frequency events.

The two lateral cameras can be rotated about their respective axes, so that the orientations of the lines of sight can either lie entirely in the poloidal plane or possess a toroidally distributed arrangement to compare in particular emission along or opposite to the plasma current direction.

Shielding is crucial to the success of this measurement. Initial tests with no shielding other than the chosen collimators quickly showed that under common TCV operating conditions detectors were swamped by highly-relativistic, uncollimated radiation. These $\gamma$-rays originate primarily from impact of runaway, highly relativistic electrons with the wall. This occurs in inductive (finiteelectric-field) discharges at low density (required for coupling of a large part of the ECRH power); these are not generally runaway discharges at all, but the minority runaway population can still generate large $\gamma$-ray showers. In the $\mathrm{MeV}$ range, these photons are not stopped by the stainlesssteel vacuum chamber and can quickly saturate the detectors. Following the initial tests, it was decided to ensure that at least $2 \mathrm{~cm}$ of tungsten were placed between any part of the vacuum chamber and any detector. The thickness was ultimately determined by space access requirements but proved to be sufficient. Some shielding is directly applied to the front of the camera in vacuum 
(Fig. 4), some outside vacuum around the port perimeter (see also Fig. 5a) [6].

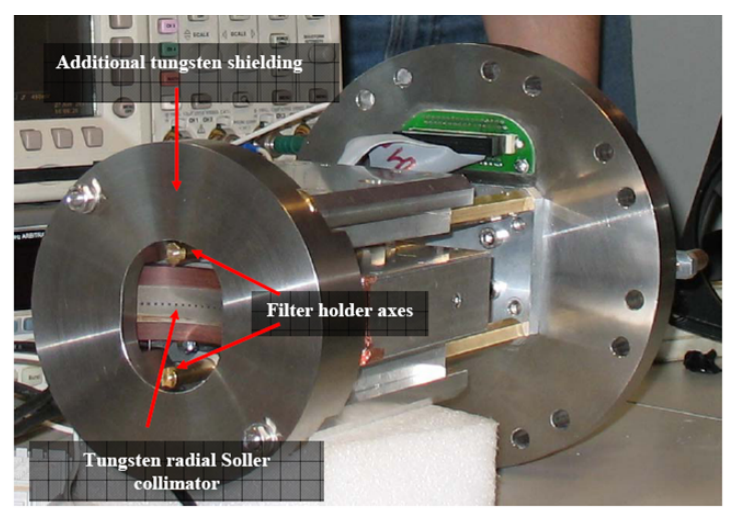

Figure 4: View of one of the cameras from the plasma side. The collimator is visible behind the tungsten shielding (additional shielding exists around the vacuum port). The electronics are housed in the aluminum box seen behind the collimator, and the (SCSI) connector feedthrough is on the main support flange.

controlled remotely through pneumatic actuators.

Finally, front-end, high-pass filtering options must be available to reduce the signal below the pile-up limit in case of exceptionally high fluence (high-power ECRH, strong MHD activity). The vacuum interface is a $0.127 \mathrm{~mm}$ thick Be window, essentially negligible for filtering purposes (except for cutting UV radiation that could contribute to noise). For maximum flexibility, two rotating filter holders, with 6 filters each (made of stainless steel and aluminum), were placed in front of the detectors. This provides a total of 36 filter combinations. The filter holders are seen in the schematic view in Fig. 5a, while the response function of a subset of filter combinations is plotted in Fig. 5b. In the final arrangement, all the filter wheels will be

\section{Pulse processing and pulse recognition}

The shortness of the charge pulses and the potentially high frequency of events demand the utmost care in signal processing. The rise time depends on the photon impact location within the crystal but averages at $\sim 30 \mathrm{~ns}$. A preamplifier card was developed in-house with the express aim of fitting within the small confines of the detector array box inside the differential vacuum. The primary element is a charge amplifier with an intrinsic rise time of $3 \mathrm{~ns}$, compatible with a 50-MHz bandwidth. Additional amplification stages follow, with the purpose of raising the noiseequivalent signal above the acquisition bit noise level. The global amplifier card gain is $50 \mathrm{~V} / \mathrm{pC}$, equivalent to $\sim 3.5 \mathrm{mV} / \mathrm{keV}$. Keeping the noise contained is crucial to the success of the assembly. The components chosen, along with a differential-output arrangement, result in a noise level of $\sim 0.2 \mathrm{fC}$, equivalent to $\sim 3 \mathrm{keV}$, comfortably below the energy resolution of the system.

Storing the full pulse history results in very large data files for each plasma discharge. This is a small price to pay in view of today's storage capacity and speed of data transfer. The advantage of being able to comfortably post-process the data, using different methodologies if necessary, can hardly be underestimated. It is nevertheless essential in practice to develop a reliable and robust method of spectroscopic analysis that can be used routinely. An extensive study of pulse recognition and pulse height analysis techniques was carried out for this purpose [12]. This encompassed a wide variety of techniques, primarily differing in the area of signal treatment (filtering). Finite impulse response (FIR) filters, particularly ones that are specifically optimized for the conditions of the measurements, have emerged as clear winners in terms of a variety of figures of merit (detection efficiency, false detections, noise sensitivity and energy accuracy). The efficient implementation of an optimized FIR filter was found to be compatible with possible future real-time applications. 

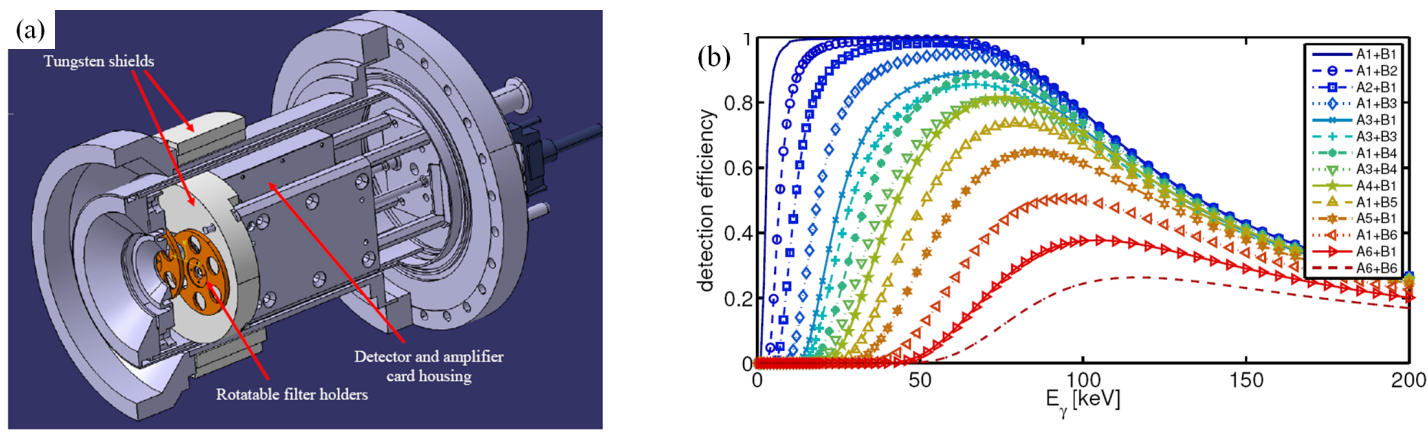

Figure 5: (a) Schematics of one of the camera assemblies within the vessel vacuum port, showing the two tungsten shields, the rotatable filter holders and the electronics box. (b) Attenuation factor vs energy for a variety of available filter combinations.

\section{Summary and conclusions}

A new, state-of-the-art tomographic HXR spectrometer has been developed for the TCV tokamak. This system features a broad dynamic range compatible with high count rates, a broad energy range and excellent $\gamma$-ray rejection through massive shielding facilitated by its compactness. Its compact design can easily be adapted or even directly ported to other devices. A host of results have already been obtained with this system $[8,13,14]$, including a study of the $2 \mathrm{D}$ poloidal distribution of HXR emissivity, a study of toroidal asymmetries in the presence of ECCD, and a study of the interaction of suprathermal electrons with various MHD modes. In all cases comparisons with numerical modeling have been performed.

\section{Acknowledgments}

This work was supported in part by the Swiss National Science Foundation.

\section{References}

[1] S. Coda, Rev. Sci. Instrum. 79 (2008) 10F501.

[2] F. Hofmann et al, Plasma Phys. Control. Fusion 36 (1994) B277.

[3] T.P. Goodman and the TCV team, Nucl. Fusion 48 (2008) 054011.

[4] Y. Peysson, S. Coda and F. Imbeaux, Nucl. Instr. and Methods in Phys. Res. A 458 (2001) 269.

[5] S. Gnesin, S. Coda, J. Decker and Y. Peysson, Rev. Sci. Instrum. 79 (2008) 10 F504.

[6] S. Gnesin, Ph.D. Thesis EPFL n. 5181 (2011).

[7] J. Kamleitner et al, 39th EPS Conf. (Stockholm, 2012), Europhys. Conf. Abstr. 36F (2012) P-1.010.

[8] J. Kamleitner, Ph.D. Thesis EPFL n. 6523 (2015).

[9] S. Gnesin and S. Coda, Int. Conf. on Burning Plasma Diagnostics (Varenna, 2008), AIP Conf. Proc. 988 (2008) 222.

[10] J. Decker and Y. Peysson, Rep. EUR-CEA-FC-1736 (2004).

[11] Y. Peysson and J. Decker, Phys. Plasmas 15 (2008) 092509.

[12] J. Kamleitner et al, Nucl. Instr. and Methods in Phys. Res. A 736 (2014) 88.

[13] J. Kamleitner et al, 40th EPS Conf. (Espoo, 2013), Europhys. Conf. Abstr. 37D (2013) P-5.127.

[14] J. Kamleitner et al, submitted to Plasma Phys. Control. Fusion (2015). 